\title{
Detection of a Theileria species in dogs in South Africa
}

\author{
P.T. Matjila ${ }^{\text {a,* }}$, A.L. Leisewitz ${ }^{\text {a }}$, M.C. Oosthuizen ${ }^{\text {a }}$, F. Jongejan ${ }^{\text {a,b }}$, B.L. Penzhorn ${ }^{\text {a }}$ \\ ${ }^{a}$ Department of Veterinary Tropical Diseases, Faculty of Veterinary Science, University of Pretoria, Private Bag x04, \\ Onderstepoort 0110, South Africa \\ ${ }^{\mathrm{b}}$ Utrecht Centre for Tick-borne Diseases (UCTD), Department of Infectious Diseases and Immunology, \\ Faculty of Veterinary Medicine, Utrecht University, Yalelaan 1, 3584 CL Utrecht, The Netherlands
}

Received 31 January 2008; received in revised form 19 June 2008; accepted 26 June 2008

\begin{abstract}
A Theileria species was detected by PCR in blood samples collected from dogs in the Pietermaritzburg area and was also found in dogs presented at the Outpatients Clinic of the Onderstepoort Veterinary Academic Hospital (OVAH), in the Pretoria area, South Africa. In the Pietermaritzburg area, 79 of the 192 samples were positive, while 3 out of 1137 of the Onderstepoort samples were positive. Three positive samples from Pietermaritzburg were co-infected with Ehrlichia canis. PCR positive samples were further analysed by the Reverse Line Blot (RLB) and sequence analysis. Phylogenetic analysis of the 18S rRNA full-length gene sequences of one sample (VT12) from Pietermaritzburg and two samples from OVAH (BC281 and BC295) revealed a close relationship with sequences of Theileria species (sable). Clinical signs of the dogs that were examined at Pietermaritzburg and OVAH included an immune-mediated condition with severe thrombocytopenia. These findings identify a Theileria sp. in dogs for the first time in South Africa and add yet another microorganism to the growing list of haemoprotozoan parasites infecting dogs worldwide. The clinical significance of this infection in dogs is poorly resolved.
\end{abstract}

(C) 2008 Elsevier B.V. All rights reserved.

Keywords: Theileria sp.; Dogs; Thrombocytopenia; South Africa

\section{Introduction}

Canine babesiosis, a haemolytic disease of significant economic importance, is the most frequently encountered tick-borne protozoal infection of dogs in South Africa (Shakespeare, 1995; Collett, 2000). The parasites associated with canine babesiosis in South Africa are Babesia rossi and Babesia vogeli (Matjila et al., 2004). B. rossi, which causes a severe disease that can be lifethreatening, is the most prevalent species isolated from

\footnotetext{
* Corresponding author. Tel.: +27 12 5298424; fax: +27 125298312 .

E-mail address: tshepo.matjila@up.ac.za (P.T. Matjila).
}

dogs presented at the Onderstepoort Veterinary Academic Hospital (OVAH) (Böhm et al., 2006). The clinical signs and pathology of the disease may include pyrexia, splenomegaly, anaemia, haemolysis and haemoglobinuria, icterus, circulatory collapse, multiple organ failure and neurological signs (Jacobson and Clark, 1994). The clinical signs of infection caused by $B$. vogeli infection has not been well documented in South Africa (Böhm et al., 2006), although B. vogeli has been detected in dogs diagnosed with clinical babesiosis presented at the Outpatients Clinic, OVAH (Böhm et al., 2006). Elsewhere B. vogeli infections have been reported to cause only a mild disease in dogs (Uilenberg et al., 1989).

Recent publications have reported on previously unknown pathogens that infect dogs and cause a 
haemolytic syndrome. Firstly, a novel large Babesia sp. has been identified in dogs in North America (Birkenheuer et al., 2004). The parasite identified was isolated from the bone marrow as well as the blood of a dog with haematological abnormalities consistent with babesiosis (Birkenheuer et al., 2004). Secondly, Rangelia vitally, a blood parasite causing a disease characterized by anaemia, jaundice, fever, splenomegaly, lymphadenopathy, haemorrhage in the gastrointestinal tract and persistent bleeding from the nose, has been described in Brazil (Loretti and Barros, 2005). $R$. vitally is suspected to be tick-transmitted and the authors have stated that the parasite is a protozoan of the phylum Apicomplexa, although different from Babesia, since it has an intra-endothelial stage. These authors did not report on any molecular comparisons, which limited determination of the phylogenetic relationship to other blood protozoan parasites. Thirdly, small babesias with similar morphology to B. gibsoni have also been described (Kjemtrup et al., 2000, 2006). Although similar in morphology to B. gibsoni, these parasites are genetically distinct and include an Asian isolate, a Spanish isolate and a Californian isolate (Kjemtrup et al., 2000). Recent molecular research has shown that the Californian isolate is genotypically and phenotypically different from the $B$. gibsoni group, and has thus been named Babesia conradae (Kjemtrup et al., 2006).

There have been no reports of pathogenic Theileria species in dogs. The only species associated with a haemolytic disease of dogs is the Babesia microti-like, controversially named parasite, Theileria annae (Zahler et al., 2000; Camacho et al., 2001, 2004; Camacho Garcia, 2006). Morphologically, this parasite has been described as a small piroplasm (Camacho Garcia, 2006). Molecular analysis of the 18S rRNA gene has shown that this parasite is closely related to $B$. microti, a rodent parasite (Zahler et al., 2000; Criado-Fornelio et al., 2003a; Conrad et al., 2006). Several authors have cited Goethert and Telford (2003) when referring to this parasite as Babesia annae. Although Goethert and Telford (2003) did not propose the name B. annae, they questioned the use of Theileria as a genus name since no evidence was presented by Zahler et al. (2000) for a preerythrocytic or lymphocyte-infecting stage, nor was there any evidence for the absence of transovarial transmission in ticks (Goethert and Telford, 2003). The provisional assignment to the genus Theileria reflects a controversial argument by some parasitologists working with piroplasms that the small Babesia should be removed from the genus Babesia (Guitián et al., 2003). In the context of our report we will, therefore, refer to the said parasite as T. annae.
Other Theileria sp. that have been reported from dogs are Theileria annulata (Criado et al., 2006) and Theileria equi (Criado-Fornelio et al., 2003b). Theileria annulata was detected from an asymptomatic dog (Criado et al., 2006), whereas T. equi was detected from three asymptomatic dogs and one symptomatic dog (Criado-Fornelio et al., 2003c). These findings were followed up, however, and as far as we know none of these Theileria parasites have subsequently been isolated from clinically reacting dogs. Our report describes a Theileria sp. isolated from dogs originating from two localities in South Africa, namely Pietermaritzburg (KwaZulu-Natal) and the Onderstepoort district of Pretoria (Gauteng). The Theileria sp. was first detected from samples collected in Pietermaritzburg in 2004. The DNA of this organism was later also detected in two clinical samples collected from two dogs presented at the OVAH in 2005. The same DNA as this organism was detected in a third clinical sample, collected from a dog presented at the OVAH in January 2007.

\section{Materials and methods}

Blood samples $(n=192)$ were collected monthly over a six-month period from the Pietermaritzburg area, during the early summer months of 2004, and late summer months of 2005 . The samples were collected routinely from dogs involved in a study of tick-repellent impregnated dog collars. Blood samples $(n=1137)$ were collected from dogs presented at the OVAH from January 2002 to January 2007. Blood-smear examinations were done by the attending clinicians on all samples. Blood samples were then collected into EDTA Vacutainer $^{\mathrm{B}}$ (Franklin Lakes, USA) tubes and sent to the Department of Veterinary Tropical Diseases, Faculty of Veterinary Science, University of Pretoria, for molecular analysis.

DNA was extracted from $200 \mu \mathrm{l}$ of each blood sample using the QIAmp ${ }^{\mathbb{R}}$ blood and tissue extraction kit (Qiagen, Hilden, Germany), following the manufacturer's protocols. PCR was performed with primers RLB-F2 (5'-GAC ACA GGG AGG TAG TGA CAA G$3^{\prime}$ ) and RLB-R2 (biotin-5'-CTA AGA ATT TCA CCT CTG ACA GT- $3^{\prime}$ ) amplifying a fragment of 460-540 bp from the 18S rRNA gene spanning the V4 region (Gubbels et al., 1999; Matjila et al., 2004). The conditions for the PCR included an initial step of $3 \mathrm{~min}$ at $37{ }^{\circ} \mathrm{C}, 10 \mathrm{~min}$ at $94{ }^{\circ} \mathrm{C}, 10$ cycles of $94{ }^{\circ} \mathrm{C}(20 \mathrm{~s})-$ $67{ }^{\circ} \mathrm{C}(30 \mathrm{~s})-72{ }^{\circ} \mathrm{C}(30 \mathrm{~s})$, with lowering of annealing step after every second cycle by $2{ }^{\circ} \mathrm{C}$ (touchdown PCR). The reaction was then followed by 40 cycles of 
denaturation at $94{ }^{\circ} \mathrm{C}$ for $30 \mathrm{~s}$, annealing at $57{ }^{\circ} \mathrm{C}$ for $30 \mathrm{~s}$ and extension at $72{ }^{\circ} \mathrm{C}$ for $30 \mathrm{~s}$. PCR-amplified products were tested with the RLB, as previously described (Matjila et al., 2004). An additional plasmid control was used as an internal positive control to ensure that all Babesia species-specific probes were correctly bound to the RLB membrane and that they were functional (Matjila et al., 2005).

PCR products that did not hybridize to any of the species-specific probes but hybridized to the Theileria genus-specific probe were selected from the samples collected in Pietermaritzburg and Onderstepoort. The RLB was repeated using a new membrane which included Theileria probes described by Nijhof et al. (2005). Samples, VT4, VT9, VT12 and VT17 collected from Pietermaritzburg and samples BC285 and BC295 and BC610 collected from the OVAH were partially sequenced (400-540 bp) using primers RLB F2 and RLB R2. These samples were selected for sequencing based on the quality and quantity of their genomic DNA. A BLAST search was performed with the obtained sequences using the BLASTn algorithm and compared with sequences deposited in GenBank. The full-length 18S rRNA gene of sample VT12 (Pietermaritzburg) and the two clinical samples, BC281 and BC295 (OVAH) were amplified using 20 pmol of primers Nbab 1F (5'-AAG CCA TGC ATG TCT AAG TAT AAG CTT TT- $\left.{ }^{\prime}\right)$ and TB Rev (5'-AAT AAT TCA CCG GAT CAC TCG- $3^{\prime}$ ) to give a PCR amplicon of $c a$ 1800 base pairs that was subsequently visualized by gel electrophoresis.

These PCR products were purified with the QIAmp ${ }^{\mathbb{R}}$ PCR purification kit (Qiagen, Hilden, Germany), and sent for sequencing at the Genetics Section of the Faculty of Veterinary Science. The full-length $18 \mathrm{~S}$ rRNA gene was sequenced in parts using 3.2 pmol of the following primers: Nbab1F $\left(5^{\prime}\right.$-AAG CCA TGC ATG TCT AAG TAT AAG CTT TT-3') (Oosthuizen et al., 2008), TB Rev (5'-AATAATTCACCGGATCACTCG-3'), BT 2R (5'-CCC GTG TTG AGT CAA ATT AAG CCG-3'), BT 3F (5'-GGG CAT TCG TAT TTA ACT GTC AGA GG-3'), (Oosthuizen et al., 2008), Nbab 4F (5'-CCG TTA ACG GAA CGA GAC CTT
AAC C-3') and Nbab 4R (5'-GGT AGG CCA ATA CCC TAC CG-3').

DNA amplicons of sample VT12, BC281 and BC295 were also cloned into the pGem Teasy vector (Promega, Leiden, The Netherlands) following the manufacturer's instructions. Twelve clones of each sample containing the amplified product were then sequenced using primers SP6 (5'-TAA ATC CAC TGT GAT ATC TTA TG- $\left.3^{\prime}\right)$ and T7 (5'-TAT GCT GAG TGA TAT CCC GCT- $\left.3^{\prime}\right)$. Sequence data for the full-length $18 \mathrm{~S}$ rRNA gene were assembled and edited to a total length of $1627 \mathrm{bp}$ using GAP 4 of the Staden package (Version 1.6.0 for Windows) (Bonfield et al., 1995; Staden, 1996; Staden et al., 2000), and deposited in GenBank. The sequences were aligned with sequences of related genera using ClustalX (Version 1.81 for Windows). The alignment was manually truncated to the size of the smallest sequence ( 1368 bp). The two-parameter model of Kimura and the Jukes and Cantor correction model for multiple base changes were used to construct similarity matrices (Jukes and Cantor, 1969; Kimura, 1980). Neighbor-joining (Saitou and Nei, 1987) and the maximum parsimony methods were used for the construction of phylogenetic trees using the Mega 3.0 software package (Kumar et al., 2004). The methods above were used in combination with the bootstrap method (Felsenstein, 1985)(1000 replicates/tree for distance methods and 100 replicates/tree for parsimony methods).

\section{Results}

Some of the processed samples were negative on blood-smear examination for piroplasms, but were suspected to be Babesia positive. Initial processing of blood samples using the RLB assay revealed that 76 of the 192 blood samples from Pietermaritzburg were positive for a Theileria sp. by hybridizing with a Theileria/Babesia genus-specific catchall probe as well as the Theileria genus-specific catchall probe (Table 1). Three of the 1137 samples collected from the OVAH, were positive for a Theileria sp. by also hybridizing with the same Theileria/Babesia genus-specific probe

Table 1

Reverse line blot hybridization results of dogs positive for only Theileria sp. and for mixed infections of Theileria sp. and E. canis

\begin{tabular}{llll}
\hline Location & $\begin{array}{l}\text { Total number of } \\
\text { collected samples }\end{array}$ & $\begin{array}{l}\text { Number of samples } \\
\text { positive for Theileria sp. }\end{array}$ & $\begin{array}{l}\text { Number of samples positive } \\
\text { for Theileria sp. and E. canis }\end{array}$ \\
\hline Pietermaritzburg & 192 & 76 & 3 \\
OVAH & 1137 & 3 & -
\end{tabular}

NB: Samples from both localities were positive for other important blood parasite. The results of these are reported in a separate manuscript. 
as well as the Theileria genus-specific catchall probe. Selection and partial sequencing (400-500 bp) of samples VT4, 9, 12 and 17 from Pietermaritzburg and samples BC281, 295 and 610 from OVAH revealed that the samples were similar to the previously described Theileria sp. characterized from sable antelope (Hippotragus niger) (Stoltsz and Dunsterville, 1992). Repeated testing of all samples on the RLB membrane that had species-specific probes that included Theileria sp. (greater kudu), Theileria sp. (grey duiker), Theileria sp. (sable) (Nijhof et al., 2005) and T. annae (CCG AAC GTA ATT TTA TTG ATT TG)

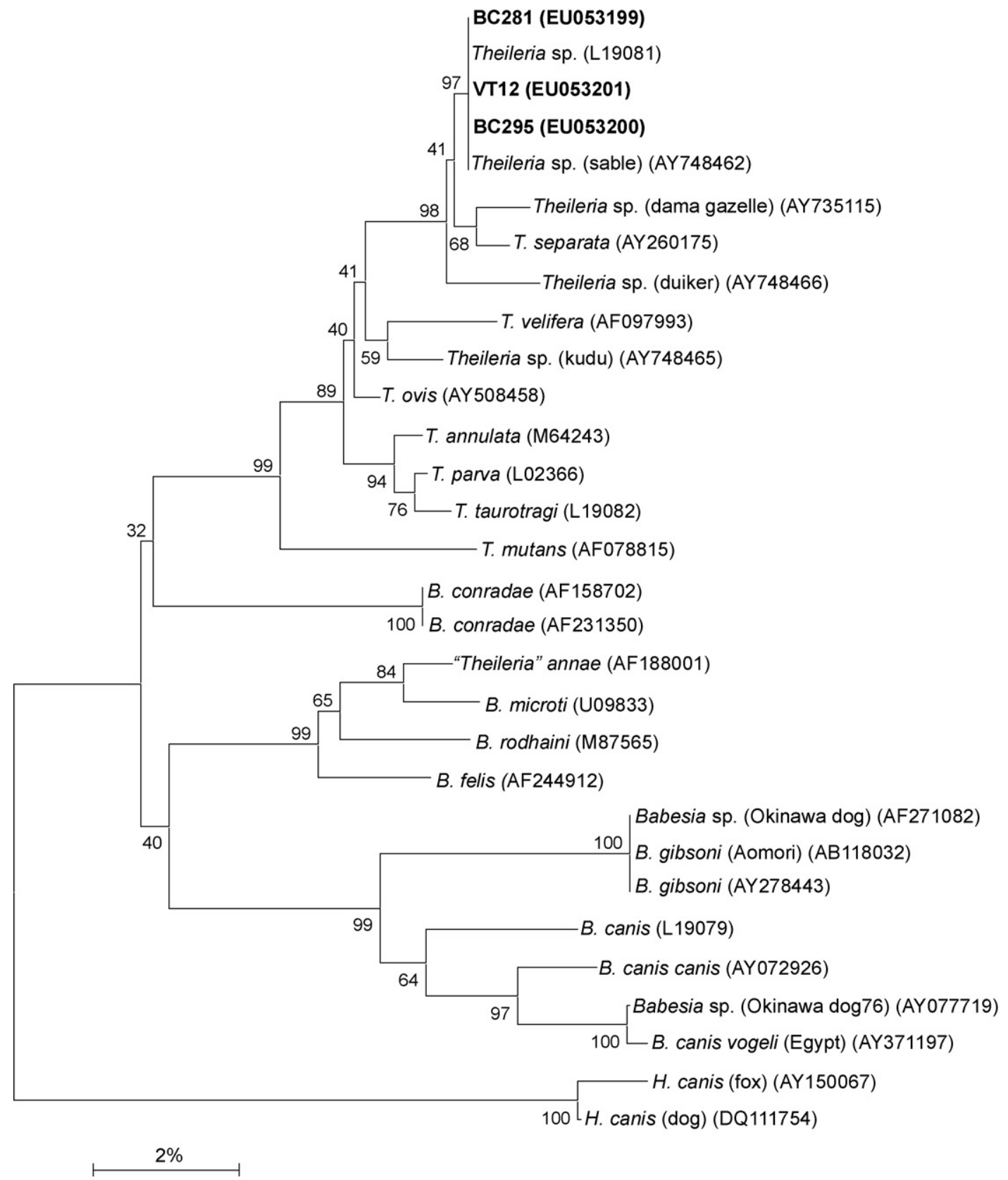

Fig. 1. Neighbor-joining tree, with the Kimura two-parameter distance (Kimura, 1980) calculation showing the phylogenetic relationship of BC281, 295 and VT12 to related species based on the 18S rRNA gene sequences. Relationships are presented as an unrooted tree with branch lengths being proportional to the estimated genetic distance between the strains. The scale bar represents the \% nucleotide difference. The GenBank accession numbers are indicated in parentheses. 
revealed that all the previously Theileria genus-specific positive samples hybridized with the Theileria sp. (sable) probe. Three further blood samples from Pietermaritzburg were concurrently infected with Theileria sp. and Ehrlichia canis, as detected by the RLB. Blood-smear examinations of Pietermaritzburg and OVAH samples did not contain any Theileriainfected leukocytes and/or red blood cells, but there were other important haemoparasites (including $B$. rossi, E. canis and mixed infections of $B$. rossi and E. canis) of dogs detected in blood samples by light microscopy and/or PCR/RLB, collected from Pietermaritzburg and from OVAH (Matjila et al., 2008).

Full-length 18S rRNA gene sequences of samples VT12 (EU053201) from Pietermaritzburg and two samples from OVAH BC281 (EU053199) and BC295 (EU053200) were compared with sequences of related genera. The BLAST search revealed highest similarities ( $99 \%$ ) with a Theileria sp. (AY748462) isolated from a sable antelope originating from Malelane (southern Kruger National Park area of South Africa), and a Theileria sp. (L19081) that was also isolated from a sable antelope and later described and named: Theileria sp. (sable) (Allsopp et al., 1994). Samples VT12, BC281 and 295 also showed $98 \%$ similarity with two Theileria sp. isolated from Texas (USA) dama gazelle (AY735116 and AY735115) and with Theileria separata (AY260175). These similarities were confirmed by both neighbor-joining and maximum parsimony phylogenetic approaches. No significant changes in the topology of the trees, or in the bootstrap values, were found when using any of the phylogenetic analysis procedures. The representative tree obtained by the neighbor-joining method with the Kimura two-parameter distance calculation (Kimura, 1980), is based on a $1368 \mathrm{bp}$ region of the $18 \mathrm{~S}$ rRNA gene (Fig. 1). In the aligned region, isolates VT12, BC281 and BC295 showed a one bp difference with Theileria sp. (sable) (AY748462) and four bp differences and a deletion with Theileria sp. (sable) (L19081).

\section{Discussion}

The only Theileria sp. currently known to cause disease in domestic dogs is the B. microti-like, T. annae (Zahler et al., 2000; Guitián et al., 2003; Camacho et al., 2004; Camacho Garcia, 2006), which has only been reported to occur in Spain. T. annae has been reported to cause a disease characterized by apathy, fever, and anaemia (Zahler et al., 2000). Severe regenerative anaemia and thrombocytopenia have been reported to be a constant characteristic of $T$. annae infection
(Camacho Garcia, 2006). The level of parasitaemia is also usually low and not statistically related to the severity of the anaemia or renal failure (Camacho Garcia, 2006).

In our study we used molecular techniques to identify a Theileria species of dogs associated with a haemolytic disease. No other causes of clinical signs could be identified in the affected dogs. The Pietermaritzburg samples were part of an independent study on acaricide-impregnated dog collars. This made it difficult for us to obtain the exact histories of dogs that tested positive for the Theileria sp. and/or E. canis. However, from the brief histories of samples that we received from dog samples VT5, 6, 14, 17 and 21, we gathered the following information. (1) The dog yielding sample VT5 had a history of anaemic episodes, which seemed to respond well to steroid treatment. This could be indicative of an immune-mediated disease. (2) Sample VT6 was collected from a 4-year-old dog, which had anorexia, fever, abdominal pain and respiratory difficulty. No piroplasms were seen in smear examination and the dog was suspected to have an immune-mediated condition. (3) Sample VT14 was collected from a dog with abdominal pain and suspected colitis. (4) Sample VT17 was collected from a 5-yearold dog presented with weight loss and fever. Smear examination of VT17 showed suspected Babesiainfected erythrocytes and a regenerative anaemia. However, this sample was PCR/RLB negative for Babesia. Further details were not provided. Finally (5) sample VT21 was collected from a 2-year-old emaciated dog with heavy hook-worm infection and thrombocytopenia. With the exception of VT14, findings were consistent with canine babesiosis (fever, anorexia, anaemia and thrombocytopenia) or similar to those described in dogs diagnosed with $T$. annae infection (fever, anaemia, and thrombocytopenia).

Detailed clinical histories were obtained from three Theileria-positive samples (BC281, 295 and 610) collected at the OVAH. Sample BC281 was collected from a 4-year-old Doberman Pinscher diagnosed with chronic-active necrotic superficial dermatitis and deep cellulitis of unknown cause, anaemia and severe thrombocytopenia. The dog was again seen three months later, when it was diagnosed with nasal trauma and severe thrombocytopenia. PCR/RLB analysis of the blood sample revealed that the dog was infected with Theileria sp. No Ehrlichia and/or Anaplasma infections were detected from sample BC281.

Sample BC295 was collected at the OVAH, from a two-and-a-half-month-old Miniature Schnauzer. On clinical examination the dog had a fever and bloody 
diarrhoea. The dog was diagnosed with parvovirus infection, based on clinical signs. PCR/RLB tests confirmed a Theileria sp. infection only. A month later, the dog was brought back to the clinic and was diagnosed with distemper and parvovirus, infection based on clinical signs. Blood samples taken on this second occasion again indicated a Theileria sp. infection by PCR/RLB tests.

Sample BC610 was collected from a dog admitted for splenomegaly diagnosed at OVAH. Haematology revealed severe thrombocytopenia and abdominal ultrasound demonstrated an enlarged spleen. The dog's condition worsened and an emergency splenectomy was performed. The thrombocyte count returned to normal the following day. It was thus suspected that the thrombocytopenia was as a result of sequestration or immune-mediated destruction of thrombocytes. PCR/ RLB tests confirmed a Theileria sp. infection and no Ehrlichia and/or Anaplasma infection. Smear examinations of the three OVAH samples (BC281, 295 and 610) did not show any piroplasms, but may have been under the detection limits for routine light microscopy as often encountered in $T$. annae infections (Camacho Garcia, 2006).

Although the pathophysiology of the detected Theileria sp. in dogs is unknown, it is apparent from the few cases described here that anaemia (possibly haemolytic), splenomegaly and a possible immunemediated syndrome may be associated with this organism. Similar clinical signs are normally seen in dogs infected with T. annae (Camacho Garcia, 2006) including haematological disorders such as thrombocytopenia, which is a common finding in the absence of Ehrlichia infection in $75 \%$ of dogs infected with $T$. annae (Camacho Garcia, 2006). Phylogenetic analysis (Fig. 1) of the Theileria sp. in dogs characterized in this study (BC281: Accession number: EUO53199; BC295: Accession number: EUO53200; and VT12: Accession number: EUO53201) showed a close similarity, with one base pair difference only, to Theileria sp. (sable) (AY748462), four base differences to Theileria sp. (sable) (L19081) and no phylogenetic relationship to $T$. annae (AF188001). Both Theileria (sable) species cause mortalities in sable antelopes (Nijhof et al., 2005). To our knowledge none of the dogs that the Theileria sp. was isolated from died as a result of the infection. As previously suggested, this may indicate evidence of a chronic established host-parasite relationship (Ebert, 1998), or it may indicate that the Theileria sp. (dog) is not as virulent in dogs as Theileria sp. (sable) is in sable antelopes (Nijhof et al., 2005). It has been shown that parasites that are known to be virulent in their typical hosts may infect incidental host without causing disease (Criado-Fornelio et al., 2003c). We can therefore currently only speculate on the clinical relevance of the detected Theileria sp. in our sampled dogs. Our findings identify a Theileria sp. in dogs for the first time in South Africa and add yet another microorganism to the list of haemoprotozoans infecting dogs. More clinical samples and data will need to be collected and analysed to understand the importance of the Theileria sp. We will therefore refer to this parasite as "Theileria sp. (dog)" which we found in South Africa.

\section{Acknowledgements}

We would like to thank all the clinicians who generously made their clinical reports available for our use (Dr M Böhm, Dr L van der Merwe, and Dr A Goddard) and Sr Riani de Kock for uploading the clinical reports. This work forms part of an ongoing $\mathrm{PhD}$ research project funded by the Thuthuka NRF fund and an institutional collaboration agreement (95401) between the Institute of Tropical Medicine, Antwerp, Belgium, and the Department of Veterinary Tropical Diseases, University of Pretoria.

\section{References}

Allsopp, M.T., Cavalier-Smith, T., De Waal, D.T., Allsopp, B.A., 1994. Phylogeny and evolution of the piroplasms. Parasitology 108, 147-152.

Birkenheuer, A.J., Neel, J., Ruslander, D., Levy, M.G., Breitschwerdt, E.B., 2004. Detection and molecular characterization of a novel large Babesia species in a dog. Vet. Parasitol. 124, 151-160.

Böhm, M., Leisewitz, A.L., Thompson, P.N., Schoeman, J.P., 2006. Capillary and venous Babesia canis rossi parasitaemias and their association with outcome of infection and circulatory compromise. Vet. Parasitol. 141, 18-29.

Bonfield, J.K., Smith, K., Staden, R., 1995. A new DNA sequence assembly program. Nucleic Acids Res. 23, 4992-4999.

Camacho, A.T., Guitian, E.J., Pallas, E., Gestal, J.J., Olmeda, A.S., Goethert, H.K., Telford III, S.R., Spielman, A., 2004. Azotemia and mortality among Babesia microti-like infected dogs. J. Vet. Intern. Med. 18, 141-146.

Camacho, A.T., Pallas, E., Gestal, J.J., Guitian, F.J., Olmeda, A.S., Goethert, H.K., Telford, S.R., 2001. Infection of dogs in north-west Spain with a Babesia microti-like agent. Vet. Rec. 149, 552-555.

Camacho Garcia, A.T.C., 2006. Piroplasma infection in dogs in northern Spain. Vet. Parasitol. 138, 97-102.

Collett, M.G., 2000. Survey of canine babesiosis in South Africa. J. S. Afr. Vet. Assoc. 71, 180-186.

Conrad, P.A., Kjemtrup, A.M., Carreno, R.A., Thomford, J., Wainwright, K., Eberhard, M., Quick, R., Telford III, S.R., Herwaldt, B.L., 2006. Description of Babesia duncani n. sp. (Apicomplexa: Babesiidae) from humans and its differentiation from other piroplasms. Int. J. Parasitol. 36, 779-789.

Criado, A., Martinez, J., Buling, A., Barba, J.C., Merino, S., Jefferies, R., Irwin, P.J., 2006. New data on epizootiology and genetics of 
piroplasms based on sequences of small ribosomal subunit and cytochrome b genes. Vet. Parasitol. 142, 238-247.

Criado-Fornelio, A., Martinez-Marcos, A., Buling-Sarana, A., BarbaCarretero, J.C., 2003a. Molecular studies on Babesia, Theileria and Hepatozoon in southern Europe. Part II. Epizootiological aspects. Vet. Parasitol. 114, 173-194.

Criado-Fornelio, A., Martinez-Marcos, A., Buling-Sarana, A., BarbaCarretero, J.C., 2003b. Molecular studies on Babesia, Theileria and Hepatozoon in southern Europe. Part I. Epizootiological aspects. Vet. Parasitol. 113, 189-201.

Criado-Fornelio, A., Martinez-Marcos, A., Buling-Sarana, A., BarbaCarretero, J.C., 2003c. Presence of Mycoplasma haemofelis, Mycoplasma haemominutum and piroplasmids in cats from southern Europe: a molecular study. Vet. Microbiol. 93, 307-317.

Ebert, D., 1998. Experimental evolution of parasites. Science (Washington) 282, 1432-1435.

Felsenstein, J., 1985. Confidence limits on phylogenies: an approach using the bootstrap. Evolution 39, 783-791.

Goethert, H.K., Telford, S.R.I., 2003. What is Babesia microti? Parasitology 127, 301-309.

Gubbels, J.M., de Vos, A.P., Van der Weide, M., Viseras, J., Schouls, L.M., De Vries, E., Jongejan, F., 1999. Simultaneous detection of bovine Theileria and Babesia species by reverse line blot hybridization. J. Clin. Microbiol. 37, 1782-1789.

Guitián, F.J., Camacho, A.T., Telford III, S.R., 2003. Case-control study of canine infection by a newly recognised Babesia microtilike piroplasm. Prev. Vet. Med. 61, 137-145.

Jacobson, L.S., Clark, I.A., 1994. The pathophysiology of canine babesiosis: new approaches to an old puzzle. J. S. Afr. Vet. Assoc. $65,134-145$.

Jukes, T.H., Cantor, C.R., 1969. Evolution of protein molecules. In: Munro, H.N. (Ed.), Mammalian Protein Metabolism. Academic Press, New York, pp. 21-132.

Kimura, M., 1980. A simple method for estimating evolutionary rates of base substitutions through comparative studies of nucleotide sequences. J. Mol. Evol. 16, 111-120.

Kjemtrup, A.M., Kocan, A.A., Whitworth, L., Meinkoth, J., Birkenheuer, A.J., Cummings, J., Boudreaux, M.K., Stockham, S.L., Irizarry-Rovira, A., Conrad, P.A., 2000. There are at least three genetically distinct small piroplasms from dogs. Int. J. Parasitol. 30, 1501-1505.

Kjemtrup, A.M., Wainwright, K., Miller, M., Penzhorn, B.L., Carreno, R.A., 2006. Babesia conradae, sp. nov., a small canine Babesia identified in California. Vet. Parasitol. 138, 103-111.
Kumar, S., Tamura, K., Nei, M., 2004. MEGA3: integrated software for molecular evolutionary genetics analysis and sequence alignment. Brief Bioinform. 5, 150-163.

Loretti, A.P., Barros, S.S., 2005. Hemorrhagic disease in dogs infected with an unclassified intraendothelial piroplasm in southern Brazil. Vet. Parasitol. 134, 193-213.

Matjila, P.T., Leisewitz, A.L., Jongejan, F., Penzhorn, B.L., 2008 Molecular detection of tick-borne protozoal and ehrlichial infections in domestic dogs in South Africa. Vet. Parasitol. 155, 152-157.

Matjila, P.T., Penzhorn, B.L., Bekker, C.P., Nijhof, A.M., Jongejan, F., 2004. Confirmation of occurrence of Babesia canis vogeli in domestic dogs in South Africa. Vet. Parasitol. 122, 119-125.

Matjila, T.P., Nijhof, A.M., Taoufik, A., Houwers, D., Teske, E., Penzhorn, B.L., De Lange, T.D., Jongejan, F., 2005. Autochthonous canine babesiosis in The Netherlands. Vet. Parasitol. 131, 23-29.

Nijhof, A.M., Pillay, V., Steyl, J., Prozesky, L., Stoltsz, W.H., Lawrence, J.A., Penzhorn, B.L., Jongejan, F., 2005. Molecular characterization of Theileria species associated with mortality in four species of African antelopes. J. Clin. Microbiol. 43, 5907-5911.

Oosthuizen, M.C., Zweygarth, E., Collins, N.E., Troskie, M., Penzhorn, B.L., 2008. Identification of a novel Babesia sp. from sable antelope (Hippotragus niger, Harris 1838). J. Clin. Microbiol. 46, 2247-2251.

Saitou, N., Nei, M., 1987. The neighbor-joining method: a new method for reconstructing phylogenetic trees. Mol. Biol. Evol. 4, 406-425.

Shakespeare, A.S., 1995. The incidence of canine babesiosis amongst sick dogs presented to the Onderstepoort Veterinary Academic Hospital. J. S. Afr. Vet. Assoc. 66, 247-250.

Staden, R., 1996. The Staden sequence analysis package. Mol. Biotechnol. 5, 233-241.

Staden, R., Beal, K.F., Bonfield, J.K., 2000. The Staden package, 1998. Methods Mol. Biol. 132, 115-130.

Stoltsz, W.H., Dunsterville, M.T., 1992. In vitro establishment and cultivation of a Cytauxzoon sp. (Theileria sp.) from a sable antelope (Hippotragus niger, Harris 1838). J. S. Afr. Vet. Assoc. 63, 182 (Abstract).

Uilenberg, G., Franssen, F.F., Perie, N.M., Spanjer, A.A., 1989. Three groups of Babesia canis distinguished and a proposal for nomenclature. Vet. Q. 11, 33-40.

Zahler, M., Rinder, H., Schein, E., Gothe, R., 2000. Detection of a new pathogenic Babesia microti-like species in dogs. Vet. Parasitol. 89, 241-248. 